\title{
Dear Doctor: A Tool to Facilitate Patient-Centered Communication
}

\author{
Aaron S. Farberg, MD ${ }^{1 \star}$, Andrew M. Lin, MD², Latoya Kuhn, MPH ${ }^{3}$, Scott A. Flanders, $\mathrm{MD}^{2}$, Christopher S. Kim, MD, MBA²
}

${ }^{1}$ Department of Surgery, University of Michigan Health System, Ann Arbor, Michigan; ${ }^{2}$ Department of Internal Medicine, University of Michigan Health System, Ann Arbor, Michigan; ${ }^{3}$ Health Services Research and Development Center of Excellence, University of Michigan, Ann Arbor, Michigan.

BACKGROUND: Patient-centered care has been identified as 1 of the 6 aims for the 21st century healthcare system. The notepad is a simple tool for reminders and personal interactions. We introduced Dear Doctor (DD) notes, a bedside notepad designed to prompt patient questions and improve patient satisfaction.

OBJECTIVE: To provide DD notes as a bedside tool to facilitate patient communication and improve patient encounters with physicians in the hospital.

DESIGN: This is a single-center, cross-sectional survey.

METHODS: Over a 3-month period (July 2009-September 2009), all hospitalized patients in the intervention group (1 general medicine and 1 cardiology unit) at a large academic medical center received a DD notepad, a pen, and instructions on its use. We surveyed patients who received the DD notes on the intervention group $(n=440)$ and compared their responses to those from a matched control group (1 general medicine and 1 cardiology unit, $\mathrm{n}=224$ ).

RESULTS: Of the 440 patients surveyed in the intervention group, $78 \%(n=343)$ received the notepads in their rooms and $47 \%$ ( $n=207)$ used them. Of the 343 patients who received the DD notepads, $65 \%(n=223)$ reported that they took notes related to their hospital stay compared to only $22 \%$ of 224 patients $(n=50)$ in the control group $(P<$ 0.001 ). The 207 patients using the DD notes had their questions more often answered by their physicians as measured on a 5-point Likert scale, compared to the control group (4.63 vs 4.45; $P<0.001$ ). However, overall rating of communication did not differ between intervention and control groups in an intention-to-treat analysis (4.55 vs $4.55, P=$ 0.89). All patients who used the DD notepads responded on the survey that communication with their physicians was enhanced to at least some degree.

CONCLUSIONS: Utilizing a bedside notepad improved patients' satisfaction with physician communication. A simple, low-cost, patient-centered tool such as the DD notes may enhance a patient's overall experience with their providers and the hospital. Journal of Hospital Medicine 2013;8:553-558. (C) 2013 Society of Hospital Medicine
In their seminal report "Crossing the Quality Chasm," the Institute of Medicine outlined patient-centered care as 1 of its 6 aims to improve the healthcare delivery system. ${ }^{1}$ Patients who are more involved in their diagnosis and treatment plan are more likely to feel respected, be satisfied with their healthcare experience, and ultimately have better outcomes. ${ }^{2,3}$ In a study of hospitalized patients, only $42 \%$ were able to state their diagnosis at the time of discharge, suggesting that hospital providers could communicate better with patients about their hospital care. ${ }^{4}$ Additionally, only $28 \%$ of hospitalized patients were able to list their medications, and only $37 \%$ were able to state the purpose of their medications. Although hospitals have taken great strides to improve the quality of patient care, publicly reported patient care surveys, such as the Hospital Consumer Assessment of Hospitals and

\footnotetext{
*Address for correspondence and reprint requests: Aaron S. Farberg, MD, Section of Plastic Surgery, Department of Surgery, University of Michigan Health System, Ann Arbor, Ml 48109; Telephone: 734-647-2892; Fax: 734-615-8401; E-mail: afarberg@med.umich.edu

Additional Supporting Information may be found in the online version of this article.

Received: March 13, 2013; Revised: June 21, 2013; Accepted: June 29, 2013

2013 Society of Hospital Medicine DOI 10.1002/jhm.2073

Published online in Wiley Online Library (Wileyonlinelibrary.com).
}

Health Systems (HCAHPS), suggest that physician communication with patients could be further improved. ${ }^{1,5}$ Furthermore, a recent report by the Institute of Medicine stresses the need to get patients and families involved in their care. ${ }^{6}$ Thus, hospital-based providers should seek to enhance the quality of their communication with patients.

With greater emphasis placed on patient- and familycentered care at many health systems, simple and easyto-implement strategies to improve communication with patients need to be developed and tested. ${ }^{7,8}$ Patients who actively participate in their healthcare by asking questions of their doctor are able to control the focus of their interaction and adjust the amount of information provided. ${ }^{9}$ Simply asking questions can have a critical impact, as 1 study found that the frequency with which patients asked questions was significantly related to the amount of information received about general and specific medical matters. ${ }^{10}$ The notepad is a common tool for reminders and personal interactions that is used in everyday life, but has not been formalized in the hospital. We introduced Dear Doctor (DD) notes, a bedside notepad designed to prompt patient questions, with the goal of facilitating patient communication with their hospitalist physicians (Figure 1). As hospitalists provide direct and indirect care to a growing number of hospitalized patients, they are likely 


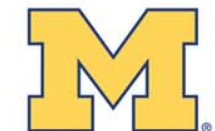

University of Michigan Health System

\section{dear doctor...}

Time:

Write questions for your healthcare here

Diagnostic \& Treatment:

(e.g. What is the reason for my hospitalization?)

Tests \& Procedures:

(e.g. What tests are planned for today?)

Medications:

(e.g. What medications will I be on?)

"I have a few more questions." (Use the back of this sheet)

FIG. 1. Dear Doctor notepad.

to be asked questions and opinions about the patients' diagnoses and plans. Furthermore, hospitalists are poised to lead institutional quality, safety, efficiency, and service improvement efforts in the inpatient setting. Becoming familiar with communication-enhancing tools, such as the DD notes, may help hospitalists in their improvement team roles.

\section{METHODS}

\section{Setting}

We conducted a study between July 2009 and September 2009 on inpatient medical wards at a large academic medical center with 610 beds and over 44,000 annual discharges. ${ }^{11}$ The internal medicine services served by attending physicians and residents comprise 
a large proportion of hospitalized patients, accounting for over 17,000 discharges per year. Each medical unit includes 32 beds.

\section{Population}

Patients over the age of 18 years admitted to a general medicine or cardiology unit and who were able to verbally communicate in English were eligible to be surveyed in the study. Patients with a length of stay $<24$ hours were excluded. A total of 664 patients were surveyed for inclusion in the study, 440 patients in the intervention group and 224 patients in the control group.

\section{Intervention}

The DD notepad included sample questions and informational prompts derived with input from a community focus group. The community focus group consisted of current and formerly hospitalized patients and family members who were asked by members of the study group what they thought would be important to include on a notepad provided to patients. From their answers the study team developed the DD notepad prototype. The DD notepad included 3 general categories of questions: (1) diagnosis and treatment, (2) tests and procedures, and (3) medications. To address other miscellaneous topics such as discharge and posthospital care needs, a section was designated for the patient to check off as "I have a few more questions (Use the back of the sheet)."

All patients admitted to the study units were intended to receive the DD notepad and pen, which were placed on the bedside table during the room change by our custodial staff. Patients who did not receive DD notepads in the intervention group during their first hospitalization day were provided with 1 by the clinical assistants working with the hospitalists. These patients did not initially receive the notepad due to logistical reasons from temporary rotating staff who were not instructed to provide the notepads. Patients were not formally prompted to use the notepad. Hospitalists, residents, and nurses on the study units were informed about the distribution of DD notes to patients on these units; however, they were not provided with any specific instructions on how they should incorporate the DD notes into their interactions with their patients. The use of the DD notepad was left to each healthcare professional's own discretion.

Members of the study team surveyed patients who had been in the hospital for a minimum of 24 hours in the intervention and control groups twice weekly. All responses were deidentified of any personal or health information. Patients were asked to rate on a scale from 1 to 5 their use of the DD notepads, their perceived value, the circumstances in which the notepads were used, and their level of satisfaction with how their physicians communicated and answered their questions $(1=$ no improvement, $5=$ significant improvement). For control patients, questions pertaining to DD notepads were not applicable and were therefore excluded.

\section{Statistical Analysis}

The data were analyzed in an intention-to-treat analysis of all 440 patients in the intervention group. Intervention and control groups were compared using $\chi^{2}$, rank sum, and Fisher exact statistical tests, with significance assigned as $P<0.05$, using SPSS software version 17.0 (SPSS, Inc., Chicago, IL). Our project was approved by the University of Michigan's institutional review board.

\section{RESULTS}

Of the 440 patients surveyed in the intervention group (1 general medicine and 1 cardiology unit), 343 (78\%) received the notepads in their rooms and 207 (47\%) used them (Figure 2). Not every patient in the intervention group received DD notepads due to inconsistent placement of DD notepads upon every room turnover. Of the patients admitted to the control group (1 general medicine and 1 cardiology unit), 224 were surveyed. Fifty-four percent of the 440 patients in the intervention group reported that they took notes related to their hospital care, compared to only $22 \%$ of the 224 patients in the control group $(P<0.001)$. Of the patients who took notes within the intervention group $(\mathrm{n}=207), 91 \%$ of them utilized the DD notepads.

Patients in the intervention group who received and used the DD notes $(n=207)$ compared to patients in

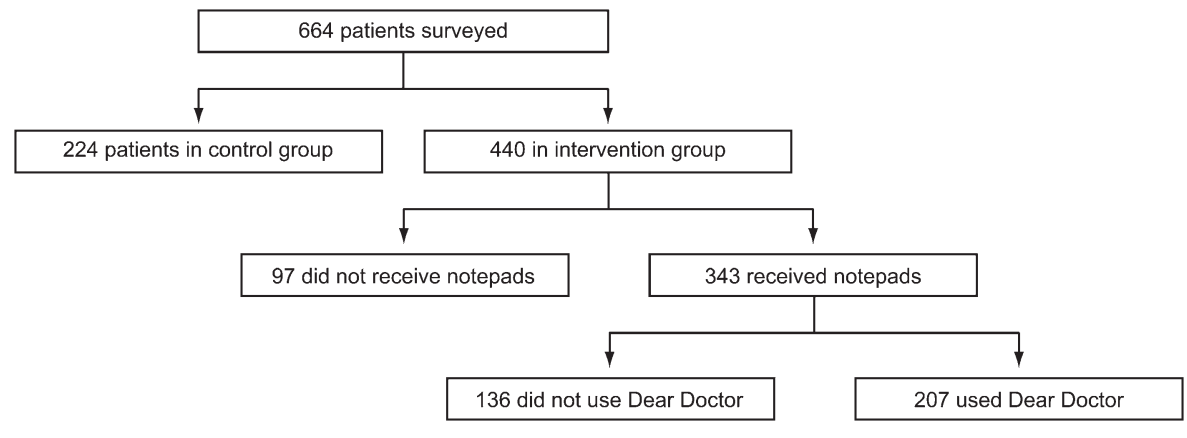

FIG. 2. Patient selection flowchart. 
the control group $(\mathrm{n}=224)$ were more likely to report that their questions were answered by their physicians $(4.63$ vs $4.45, P<0.001)$. In an intentionto-treat analysis of all 440 patients in the intervention group, the overall satisfaction with physician communication was not significantly different between the intervention and control groups as measured on a 5point Likert scale (4.55 vs $4.55, P=0.89$ ). However, $89 \%$ of the patients in the intervention group who used the notepads felt that DD notepads either moderately or significantly improved their communication with their providers (Figure 3).

When the 207 patients who received DD notepads were asked how they used this tool, $99 \%$ of these patients used DD to write down questions, $82 \%$ to keep track of tests and procedures, and $54 \%$ indicated that their family and friends also used the notepads during the hospital stay (Table 1). Among these patients who utilized the DD notepads, $93 \%$ reported that they would use them again in the future.

Of the 97 patients in the intervention group who did not receive a DD notepad, we asked if they would use the DD notepad if they were made aware of such a tool. Of these patients, $77 \%$ agreed that they would use DD notes if they were made available in the future, $100 \%$ of them said that they would use DD to write down questions, $97 \%$ indicated that they would write notes about tests and procedures, and $88 \%$ of them believed that their families and friends would use DD notes.

\section{DISCUSSION}

As hospitals place greater emphasis and value on patient-centered care as part of their clinical mission, it is important to develop tools to help facilitate the doctor-patient relationship. We found that patients who were provided the Dear Doctor notepad were more satisfied that their doctors answered their questions and felt this tool enhanced their ability to

\section{Perceived Improvement in Communication}

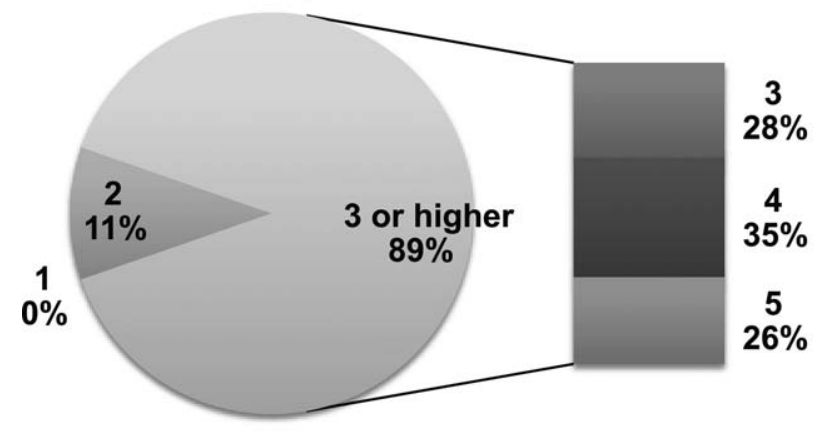

1 = no improvement; 5 = significant improvement

FIG. 3. Survey responses. The majority of patients who used Dear Doctor (DD) notes $(n=207)$ reported 3 or greater $(89 \%)$ in their perceived improvement in communication by DD notes $(1=$ no improvement, $2=$ little improvement, 3 = modest improvement, $4=$ noticeable improvement, $5=$ significant improvement). communicate with their physicians. Employing the use of a familiar tool such as the notepad to remind patients about specific issues in their interactions with their providers can be a powerful intervention. Our study demonstrated that the DD notepad was widely accepted by patients, and that almost all of them would use this tool if it were made available to them in the future.

Other tools and methods to enhance the quality of communication between patients and their healthcare providers have included using whiteboards in the patients' rooms to relay the care plan to the patients, implementing bedside rounds by the healthcare team, and multidisciplinary huddling to coordinate information to the patient. ${ }^{12,13}$ Studies of these communication tools have shown potential to improve teamwork, interaction, and patient care. All of these have their own merit and value, and our DD notepad should be considered an adjunct to existing methods to enhance the patient care experience. A bedside tool that is familiar in form to most patients also needs to have the feature of easy access and use. Once this barrier has been removed for the patient and their family members, tools such as the DD notepad can impact the patient-centeredness by fostering increased and better quality dialogue between the patients, their family members, and healthcare providers.

The DD notepad represents a means of communication that may have the potential to empower patients. It is possible that through question prompts, the DD notepad stimulates the patient to be an active partner with his or her healthcare team. This may enable patients to have some sense of control and accountability of their care in a setting where they would otherwise feel overwhelmed or powerless. The 3 general categories to help patients write down their questions included diagnosis, treatment plan, and medications. In the inpatient setting, where patient-care activities can be fast paced, and patients are unable to recall some details when speaking to the healthcare team, these notes may remind the patient to write their thoughts down so that they may be remembered for a future time. In situations where patients may not know which questions to ask, the question prompts may be particularly helpful. We did not assess whether our particular question prompts were the key elements that resulted in their perceived value, or whether simply placing a blank notepad at the bedside would also have been successful. However, the specific questions were suggested by the focus groups. Enhanced communication, focusing on the patients' understanding of their condition, and the need to pursue certain diagnostic or therapeutic interventions, may help patients to be better prepared for the next course of plan. These topics of reasons for hospitalization, treatment plan, and medication changes are also important for patients to be active participants in their care, in particular as they transition from 1 site of 
TABLE 1. Use of Dear Doctor Notepad

\begin{tabular}{|c|c|c|c|c|c|c|}
\hline & $\begin{array}{l}\text { Wrote Notes? } \\
(P<0.001)(\%)\end{array}$ & $\begin{array}{l}\text { Used DD? } \\
\text { (\%) }\end{array}$ & $\begin{array}{c}\text { Use in } \\
\text { Future? (\%) }\end{array}$ & $\begin{array}{c}\text { Frequency } \\
\text { of Questions } \\
\text { Answered } \\
(P<0.001)\end{array}$ & $\begin{array}{l}\text { DD Improved } \\
\text { Communication }\end{array}$ & $\begin{array}{l}\text { Satisfied With } \\
\text { Communication? } \\
\quad(P=0.89)\end{array}$ \\
\hline Control $(n=224)$ & 22 & - & - & 4.45 & - & 4.55 \\
\hline
\end{tabular}

care to the next, and their healthcare will be delivered by different providers.

There are several limitations to our study. First, this intervention was performed at a single hospital site with only 2 clinical services (general medicine, cardiology) represented in the study groups. Although we do not have any causal reasons to believe this tool would be looked upon differently by patients on other clinical services, it is possible that patients on a different clinical unit or service may view this tool as less or more useful. Second, as the patients were not randomized to intervention, but rather based on the units to which they were admitted, it is possible that other variables, such as the experience of the unit staff, the patient's condition, and housestaff-based service versus hospitalist-based service may have played a role in how the patients perceived the use of DD notes. Third, patients were only surveyed if they were able to verbally communicate in English. These notes may not be as useful in hospital settings to populations with language or literacy barriers. Fourth, the logistical implementation of DD notes limited our ability to deliver the DD notepads in every patient's rooms, where only $78 \%$ of the intervention group received the DD notepads. This may be the reason that we did not find that overall satisfaction with physician communication differed between intervention and control groups. Nonetheless, we performed an intention-to-treat analysis to minimize any biases in our analysis. Last, although our survey of patients asked about their satisfaction in using the DD note pads, we did not compare these results with those of Press-Ganey or HCAHPS scores of patients on the intervention group versus the control group. Additionally, lack of data about type, quality, and quantity of questions asked by a control group to see if the notepads actually improved quality of questions asked is a limitation; however, we believe our outcomes of interest were most specifically evaluated through our survey questions.

DD notes show that the majority of patients who use this tool feel a modest to significant improvement in communication with their providers. Although the quality of medical care is undoubtedly the first priority, the patients' view of their care, which includes communication, is arguably just as important. An often-forgotten goal of hospitals and clinics is to provide service excellence along with high-quality care.
Thus, it is imperative for hospitals and their care providers to not only focus on the quality and safety of the clinical care, but also be mindful of the patient's entire experience throughout their hospital stay. Many of the categories of questions asked in the HCAHPS address the patient's experience and perspectives of hospital care. Furthermore, the role of the HCAHPS survey in the Value-Based Purchasing rules may enhance the importance of these notepads. As the results of HCAHPS are becoming more transparent and available to the public, the impact of such results will have a greater significance to the future of the hospital's clinical mission.

\section{CONCLUSION}

DD notepads are a simple, low-cost, patient-centered tool that can be an effective reminder for patients to ask their healthcare providers questions related to their hospital care. Utilizing a common tool such as the notepad, redesigned for the healthcare setting, can serve to help healthcare providers interact with their patients. Patient satisfaction may be higher in patients who use the DD notepad.

Disclosures: Aaron S. Farberg, MD, and Andrew M. Lin, MD, contributed equally in every way and should be considered co-first authors. This work was supported by a University of Michigan Fostering Innovations Grant. The authors have no conflicting financial interests.

\section{References}

1. Committee on Quality of Health Care in America, Institute of Medicine. Crossing the Quality Chasm: A New Health System for the 21st Century. Washington, DC: National Academy Press; 2001.

2. Venetis MK, Robinson JD, Turkiewicz KL, Allen M. An evidence base for patient-centered cancer care: a meta-analysis of studies of observed communication between cancer specialists and their patients. Patient Educ Couns. 2009;77(3):379-383.

3. Stewart MA. Effective physician-patient communication and health outcomes: a review. Can Med Assoc J. 1995;152:1423-1433.

4. Makaryus AN, Friedman EA. Patients' understanding of their treatment plans and diagnosis at discharge. Mayo Clin Proc. 2005;80(8): 991-994.

5. Jha AK, Orav EJ, Zheng J, Epstein AM. Patients' perception of hospital care in the United States. N Engl J Med. 2008;359:1921-1931.

6. Institute of Medicine of the National Academies.Best care at lower cost: the path to continuously learning health care in America. Available at: http://www.iom.edu/Reports/2012/Best-Care-at-LowerCost-The-Path-to-Continuously-Learning-Health-Care-in-America.aspx. Accessed October 5, 2012.

7. Moore LG, Wasson JH. An introduction to technology for patientcentered collaborative care. J Ambul Care Manage. 2006;29:195-198.

8. Griffin SJ, Kinmonth A-L, Veltman MWM, Gillard S, Grant J, Stewart $M$. Effect on health-related outcome of interventions to alter the interaction between patients and practitioners: a systematic review of trials. Ann Fam Med. 2004;2(6):595-608

9. Kaplan SH, Greenfield S, Gandek B, Rogers WH, Ware JE Jr. Characteristics of physicians with participatory decision-making styles. Ann Intern Med. 1996;124(5):497-504. 
10. Street RL Jr. Information-giving consultations: the influence of patients' communicative styles and personal characteristics. Soc Sci Med. 1991:32(5):541-548.

11. University of Michigan Health System.Patient care and University of Michigan Health System. Available at: http://www.uofmhealth. org/about\%2Bumhs/about-clinical-care. Accessed August 31, 2012.
12. Singh S, Fletcher KE, Pandl GJ, et al. It's the writing on the wall: whiteboards improve inpatient satisfaction with provider communication. Am I Med Oual. 2011;26(2):127-131

13. Sehgal NL, Green A, Vidyarthi AR, Blegen MA, Wachter RM. Patient whiteboards as a communication tool in the hospital setting: a survey of practices and recommendations. J Hosp Med. 2010;5(4):234-239. 